\title{
CYC1 Silencing Sensitizes Osteosarcoma Cells to TRAIL-Induced Apoptosis
}

\author{
Guodong Lia Dong Fu ${ }^{a}$ Wenqing Liang ${ }^{b}$ Lin Fan $^{a}$ Kai Chen ${ }^{a}$ Liancheng Shan ${ }^{a, c}$ \\ Shuo Hu $\mathrm{H}^{\mathrm{a}}$ Xiaojun Ma ${ }^{\mathrm{a}}$ Ke Zhou ${ }^{\mathrm{d}}$ Biao Cheng ${ }^{\mathrm{a}}$ \\ aDepartment of Orthopedics, Tenth People's Hospital of Tongji University, Shanghai, ${ }^{b}$ Department of \\ Orthopaedics, Shaoxing People's Hospital, Shaoxing, 'Postdoctoral Research Station of Biomedical \\ Engineering, School of Life Science and Technology, Tongji University, Shanghai, dPET/CT Center, \\ Chengdu Military General Hospital Jinniu District, Chengdu, Sichuan, China
}

\section{Key Words}

Osteosarcoma $•$ Cytochrome $\mathrm{c} 1 \cdot$ Cytochrome $\mathrm{c} \cdot$ Apoptosis $\bullet$ TRAIL

\begin{abstract}
Aims: Osteosarcoma (OS) is an aggressive bone malignancy with poor prognosis. Many OS cells are resistant to apoptotic induction by tumor necrosis factor-related apoptosis inducing ligand (TRAIL). In our previous study, we found that the serum level of cytochrome c1 (CYC1) is significantly higher in OS patients than in healthy subjects. Our aim was to investigate the effects of CYC1 silencing on TRAIL-induced apoptosis in human OS in vitro and in vivo along with the underlying mechanisms. Methods: First, we determined the expression of CYC1 in human OS tumors and cell lines versus normal adjacent tissues and cell line. We then studied the effects of CYC1 silencing alone or in combination with TRAIL on OS cell growth and apoptosis in vitro and OS tumorigenesis in vivo. Results: We found that CYC1 is overexpressed in human OS tissues and cell lines. CYC1 silencing by shRNA transfection inhibits proliferation, slightly induces apoptosis in human OS cells in vitro, and suppresses human OS tumor growth in a mouse xenograft model in vivo. Additionally, CYC1 silencing sensitizes OS to TRAIL-induced apoptosis in vitro and in vivo. Our results also showed that CYC1 silencing significantly reduces complex III activity and potentiates TRAIL-induced cytochrome $\mathrm{c}$ release and caspase- 9 activation in OS cells, suggesting that $\mathrm{CYC} 1$ silencing acts via the mitochondria-dependent apoptotic pathway. Conclusion: Taken together, our results provide evidence that CYC1 plays an important role in OS tumorigenesis, and modulation of CYC1 may be an effective strategy to potentiate OS to apoptotic induction by TRAIL.
\end{abstract}

G. Li, D. Fu and W. Liang contributed equally to this work.

\section{Ke Zhou,}

and Biao Cheng
PET/CT Center, Chengdu Military General Hospital, No. 270, Rongdu Avenue, Jinniu District, Chengdu 610083, Sichuan, (China); and Department of Orthopaedic, The Tenth People's Hospital, Tongji University, Shanghai 200092 (China)

E-Mail zhouke791@163.com and E-Mail Drchengbiao@126.com 


\section{Cellular Physiology and Biochemistry}

Cell Physiol Biochem 2014;34:2070-2080

\begin{tabular}{l|l}
\hline DOI: $10.1159 / 000366402$ & (C) 2014 S. Karger AG, Basel
\end{tabular}

Publisned online: November 28, $2014 \quad$ www.karger.com/cpb

Li et al.: CYC1 Promoting Osteosarcoma Apoptosis

\section{Introduction}

Osteosarcoma (OS) is the most common malignant bone tumor affecting children and adolescents [1]. Current standard treatment is to use neoadjuvant chemotherapy (chemotherapy given before surgery) followed by surgical resection [2]. However, owing to resistant to commonly used chemotherapeutic agents [3], OS has one of the lowest survival rates for pediatric cancer, with five-year survival ranging from $65 \%$ to $75 \%$ for localized disease and $<30 \%$ for patients with metastases [4]. Therefore, new approaches need to be considered to overcome thermotherapy resistance in the treatment of OS in order to improve the clinical outcome in patients.

Tumor necrosis factor-related apoptosis inducing ligand (TRAIL) is a member of the tumor necrosis factor (TNF) family of cytokines. TRAIL activates the cell surface death receptors (DRs) DR4 and DR5 (also referred to as TRAIL-R1 and TRAIL-R2, respectively), which in turn initiate a cascade of events resulting in stimulation of the extrinsic apoptotic pathway. TRAIL exhibits selective cytotoxicity towards cancer cells; and recombinant TRAIL and agonistic TRAIL-R1/R2 monoclonal antibodies are being developed as novel anti-cancer agents for a variety of malignancies [5]. However, many osteosarcomas are resistant to TRAIL, and induction of apoptosis requires combination treatment with other chemotherapeutic agents [6, 7].

Cytochrome c1 (CYC1) is a heme-containing subunit of complex III (also referred to as cytochrome bc1 complex), which forms the third proton pump in the mitochondrial electron transport chain. CYC1 directly interacts with cytochrome c (Cytc) and mediates electron transport from cytochrome B to Cytc during oxidative phosphorylation [8]. In our previous study, we identified CYC1 as a promising biomarker for early diagnosis of OS [9]. We found that the serum level of CYC1 in OS patients is significantly higher than that in healthy subjects. Additionally, the serum level of CYC1 in OS patients exhibits a significant decrease after surgical resection. These findings suggest that CYC1 might play a role in OS tumorigenesis. In addition to OS, CYC1 has also been found to be overexpressed in nasopharyngeal carcinoma (NPC) tissues and cell lines [10].

Interestingly, a recent study reported that CYC1 cleavage by caspase-3 disrupts mitochondrial function and enhances Cytc release and apoptosis [11]. Thus, we speculated that CYC1 downregulation in OS might sensitize OS cells to apoptotic induction by TRAIL through promoting Cytc release. In this study, we investigated the effects of CYC1 silencing on OS cell growth and apoptosis in vitro and OS tumorigenesis in vivo, either alone or in combination with TRAIL.

\section{Materials and Methods}

\section{Clinical specimens}

Human OS and matched adjacent normal tissues ( $\mathrm{n}=30$ ) were obtained from patients at Shanghai Tenth People's Hospital affiliated with Tongji University. All patients provided written informed consent. The study protocol was approved by the Ethics Committee of Tongji University.

\section{Cell culture and lentirival transfection}

Human fetal osteoblastic cell line (hFOB) and the human OS cell lines HOS, 143B, and MG-63 were provided by the Chinese Academy of Medical Sciences. Cells were grown in RPMI-1640 (Invitrogen) supplemented with $10 \%$ fetal bovine serum (Gibco), $100 \mathrm{U} / \mathrm{ml}$ penicillin, and $100 \mu \mathrm{g} / \mathrm{ml}$ streptomycin. Cells were incubated at $37^{\circ} \mathrm{C}, 5 \% \mathrm{CO}_{2}$ in a humidified incubator [12].

For CYC1 silencing, we constructed a lentiviral small hairpin RNA (shRNA)-encoding system using the pHBLV-U6-Puro lentiviral RNAi vector (Hanbio, Shanghai, China). The targeting sequence of CYC1 shRNA was: 5'-GCTCTGGAGCAGTTCCGATATC-3'. The recombinant lentivirus of CYC1 shRNA was produced by cotransfection of 293T cells with plasmids PSPAX2 and PMD2G using LipoFiter (Hanbio, Shanghai, China). The lentivirus-containing supernatant was harvested $48 \mathrm{~h}$ after transfection and filtered through $0.22-\mu \mathrm{m}$ 


\section{Cellular Physiology and Biochemistry}

Cell Physiol Biochem 2014;34:2070-2080

\begin{tabular}{l|l}
\hline DOI: $10.1159 / 000366402$ & (C) 2014 S. Karger AG, Basel
\end{tabular}

Published onlıne: November 28, 2014 www.karger.com/cpb

Li et al.: CYC1 Promoting Osteosarcoma Apoptosis

Table 1. Primers used for quantitative real-time PCR

\begin{tabular}{ll}
\hline Gene & Primer \\
\hline CYC1 (Cytochrome c1) & 5'-CTCCCTGCTCACGGGCTACT-3' (forward) \\
& 5'-GGGTGCCATCGTCAAACTCTAA-3' (reverse) \\
GAPDH & 5'-GGCAAGTTCAACGGCACAG-3' (forward) \\
& 5'-CGCCAGTAGACTCCACGAC-3' (reverse) \\
\hline
\end{tabular}

cellulose acetate filters (Millipore, USA). The recombinant lentivirus was concentrated by ultracentrifugation (2 h at 50,000 $\times$ g).

To establish stable CYC1-konckdown cell lines, MG63 and 143B cells were transduced with the lentiviral RNAi vector at an MOI of approximately 10-50 in the presence of $5 \mu \mathrm{g} / \mathrm{ml}$ polybrene. After $24 \mathrm{~h}$, culture medium was removed and fresh medium was added. At $72 \mathrm{~h}$ after transduction, puromycin $(5 \mathrm{mg} /$ $\mathrm{ml}$ ) was added to the medium for stable cell line selection. The empty lentivector lenti-puromycin was used as negative control. After antibiotic selection for 3 weeks, stable CYC1-konckdown cells were obtained.

\section{RNA isolation and quantitative real-time PCR}

Total RNA was extracted using Trizol reagent (Invitrogen, USA) following manufacturer's instructions. cDNA was synthesized using PrimeScript RT reagent Kit with gDNA Eraser (Takara, Japan). Quantitative real-time PCR was performed using SYBR-Green PCR kit (Takara) on a Step one plus System (Applied biosystems).

The specific primers used for PCR are shown in Table 1. The relative mRNA expression was normalized to GAPDH. All reactions were performed in triplicate.

\section{Protein extraction and western blot analysis}

Protein from enriched fractions of mitochondria and cytosol was extracted using isotonic mitochondrial buffer (MB: $210 \mathrm{mM}$ mannitol/70 mM sucrose/1 mM EDTA/10 mM Hepes, pH 7.5) as described elsewhere [13]. Protein concentration was determined using a BCA protein assay kit (Keygen, China). Protein samples $(50 \mu \mathrm{g})$ were separated by SDS-PAGE and transferred to NC membranes (Millipore, USA). After incubation in $5 \%$ BSA/TBST for $1 \mathrm{~h}$ at room temperature to block non-specific binding, membranes were incubated with antibodies against CYC1 (Protein Tech Group, Chicago, IL, USA), PARP, cytochrome c, cytochrome c oxidase subunit 4 (COXIV), and $\beta$-actin (all from Santa Cruz) in Tris-buffered saline at $4^{\circ} \mathrm{C}$ overnight. After washing, membranes were incubated with secondary antibody for $1 \mathrm{~h}$ at room temperature. Protein bands were visualized with enhanced chemiluminescence system (Amersham, Arlington Heights, IL, USA). $\beta$-actin or COXIV was used as an internal control.

\section{Cell proliferation assay}

143B and MG-63 cells transfected with control, Lv-NC, or Lv-CYC1 shRNA were seeded in 96-well plates at $2 \times 10^{3} /$ well and cultured at $37^{\circ} \mathrm{C}, 5 \% \mathrm{CO}_{2}$ in a humidified incubator. At specific time points, medium was removed, and cells were incubated with MTT (Sigma,USA) at a final concentration of $5 \mathrm{mg} / \mathrm{ml}$ for $4 \mathrm{~h}$. The reaction was stopped by addition of $150 \mu \mathrm{D}$ DSO and the optical density at $570 \mathrm{~nm}$ was determined on a microplate reader (Molecular Devices, CA, USA).

\section{Cell apoptosis assay}

Cell apoptosis was determined using the ApoAlert Annexin V kit (Clontech, Mountain View, CA, USA) following manufacturer's instructions. 143B and MG-63 cells transfected with control, Lv-NC, or LvCYC1 shRNA were seeded in six-well plates at $2 \times 10^{4} /$ well and cultured for $24 \mathrm{~h}$. Cells were subsequently incubated with TRAIL $(1,10,50,100 \mathrm{ng} / \mathrm{ml})$ for $72 \mathrm{~h}$. After treatment was completed, cells were harvested, stained with annexin V-FITC and propidium iodide (PI), and analyzed on a FASC flow cytometer (San Diego, CA).

\section{Complex III activity}

143B and MG-63 cells transfected with control, Lv-NC, or Lv-CYC1 shRNA were seeded in six-well plates at $1 \times 10^{5} /$ well for $3 \mathrm{~d}$. The activity of Complex III was measured by following increase in absorbance due to the reduction of cytochrome $\mathrm{c}$ at $550 \mathrm{~nm}$ using the Complex III activity quantitative assay kit (Genmed, Shanghai, China) following manufacturer's instructions. 


\section{Cellular Physiology and Biochemistry}

Cell Physiol Biochem 2014;34:2070-2080

\begin{tabular}{l|l}
\hline DOI: $10.1159 / 000366402$ & (c) 2014 S. Karger AG, Basel
\end{tabular}

Published online: November 28, 2014

www.karger.com/cpb

Caspase 9 activity

Activity of Caspase9 was measured using Caspase 9 activity assay kit (Beyotime, China) following Vendor's protocol. Caspase activity assay was based on the ability of the active enzyme to cleave the chromophore from caspase-9 substrate Ac-LEHD-pNA to p-nitroanilide (pNA) [14]. The release of pNA was measured at $405 \mathrm{~nm}$ in a microplate reader. Results are presented as the relative change in activity compared to the control.

\section{In vivo tumorigenesis}

6-Week-old male nu/nu nude mice were housed under pathogen-free conditions. 143B cells transfected with control, Lv-NC, or Lv-CYC1 shRNA were cultured to near confluence, harvested, and resuspended in PBS at $1 \times 10^{7}$ cells $/ \mathrm{ml}$. Cells $(0.1 \mathrm{ml})$ were then injected subcutaneously into the flank of nude mice. Mice received a single intravenous injection of $10 \mathrm{mg} / \mathrm{kg}$ TRAIL (six in each treatment group) every three days for six weeks. The tumor size was measured every week using a caliper. The tumor volume was calculated using the formula $1 / 6$ pab2 ( $\mathrm{p}=3.14$; $\mathrm{a}$, long-axis diameter of the tumor; $\mathrm{b}$, short-axis diameter of the tumor). Growth curves were plotted from the tumor volume (means \pm SD) in each treatment group. At the end of the 6-week treatment, the animals were sacrificed under anesthesia and tumors were immediately harvested, measured, and weighed.

Tumor tissue analysis

After the tumors $(n=6)$ were harvested, tumor tissues were immediately fixed in paraformaldehyde at room temperature for $48 \mathrm{~h}$ and embedded in paraffin. The nuclei with fragmented DNA were detected by the terminal deoxyribonucleotidyl transferase (TDT)-mediated dUTP-digoxigenin nick end labeling (TUNEL) assay using an in situ cell death detection kit (Roche Diagnostics, Mannheim, Germany) according to manufacturer's protocol.

\section{Statistical analysis}

Data are expressed as means \pm SD. Multiple comparisons were made using one-way analysis of variance followed by Fisher's tests. Differences with a $\mathrm{p}<0.05$ were considered statistically significant.

\section{Results}

CYC1 is overexpressed in human OS tissues and cell lines

It has been reported that the serum CYC1 level in OS patients is significantly higher than that in healthy subjects [9]. In this study, we assessed the mRNA expression of CYC1 in 30 human OS tissues vs. matched adjacent normal tissues using quantitative real-time PCR. We found that the CYC1 mRNA level in OS tissues was significantly higher than that in adjacent normal tissues (Fig. 1A). We then determined the mRNA and protein expression of CYC1 in the human fetal osteoblastic cell line hFOB and the human OS cell lines HOS, 143B, and MG-63, using quantitative real-time PCR and western blot analysis, respectively. Our results showed that the mRNA and protein levels of CYC1 were significantly higher in the OS cell lines than in the normal osteoblastic cell line (Fig. 1B, 1C). Therefore, CYC1 is overexpressed in human OS tissues and cell lines in vivo and in vitro. These findings, along with previous reports [9] suggest that CYC1 might play a role in OS tumorigenesis.

\section{CYC1 silencing inhibits growth and slightly induces apoptosis in OS cells}

We then investigated the effects of CYC1 silencing on 143B and MG-63 cell growth and apoptosis. First, we confirmed that Lv-CYC1 shRNA transfection effectively reduced the mRNA and protein expression of CYC1 in 143B and MG-63 cells (Fig. 2A). We then studied cell proliferation using the MTT assay, and cell apoptosis using annexin V-FITC/PI-double staining flow cytometry. Our data showed that Lv-CYC1 shRNA transfection significantly inhibited growth in 143B and MG-63 cells compared with control or Lv-NC transfection (Fig. 2B). Although the increase of apoptosis in CYC1-silenced cells was statistical significant compared with control (Fig. 2C), it was actually slight $(1.2 \pm 0.6 \%$ to $2.9 \pm 0.7 \%$ for $143 \mathrm{~B}$ cells 


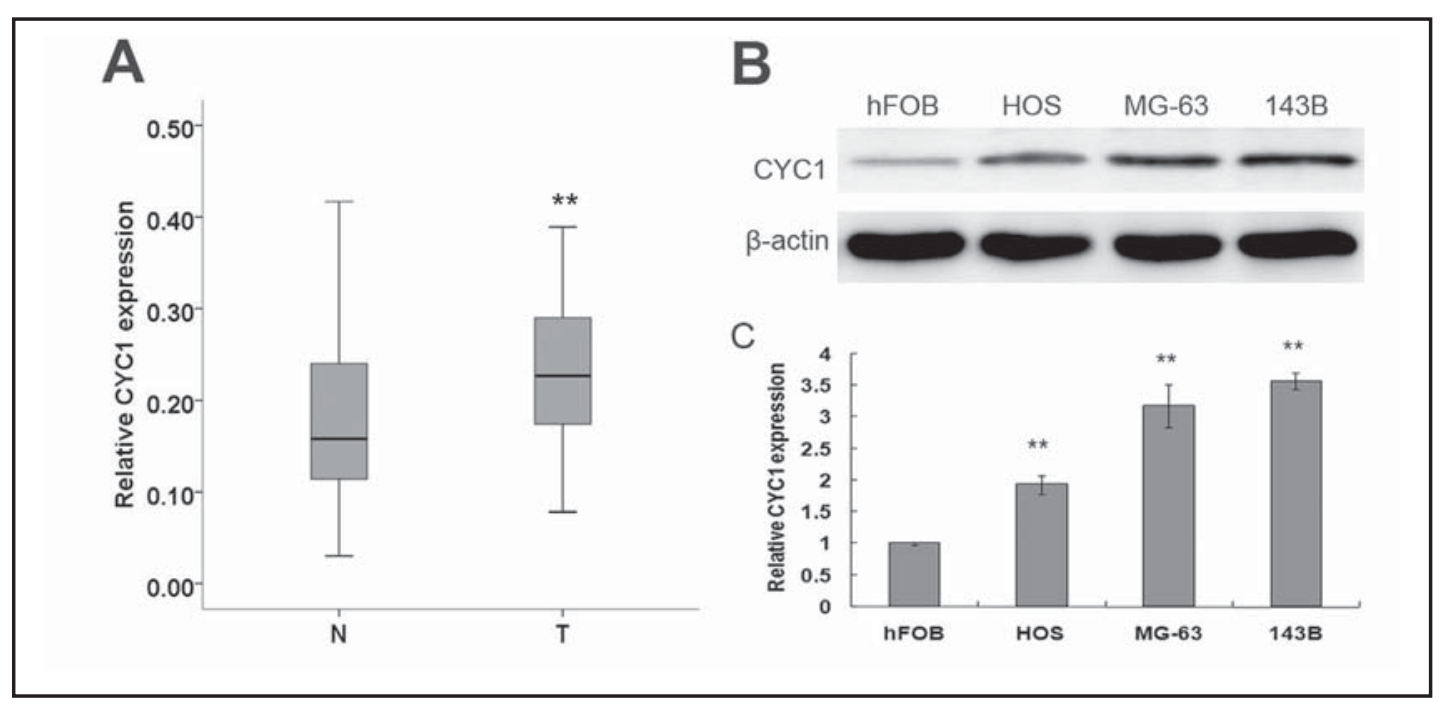

Fig. 1. CYC1 is overexpressed in human OS tissues and cell lines. (A) The mRNA expression of CYC1 in human OS and adjacent normal tissues by real-time PCR $(n=30)$. Data were normalized to GAPDH. (B) The protein expression of CYC1 in human fetal osteoblastic cell line (hFOB) and the human OS cell lines HOS, MG-63, and 143B by western blot analysis. (C) The mRNA expression of CYC1 in hFOB, HOS, MG-63, and 143B cell lines by real-time PCR. Data are expressed as means \pm SD. ${ }^{*} p<0.05,{ }^{* *} p<0.01$ compared with normal tissues or hFOB.

and $1.7 \pm 0.4 \%$ to $2.7 \pm 0.3 \%$ for MG-63 cells). We then examined the level of cleaved PARP. PARP is a family of proteins mainly involved in DNA repair and programmed cell death. Proteolytic cleavage of PARP by caspases is considered to be a hallmark of caspase activation and apoptosis. We found that CYC1 silencing slightly increased levels of cleaved PARP (Fig. 2D). We also examined complex III activity, because CYC1 is an essential constituent of complex III, a central component of the mitochondrial energy production machinery. Our data showed that in 143B and MG-63 cells transfected with Lv-CYC1 shRNA, the complex III activity was just about $50 \%$ of that of Lv-NC or control-transfected cells (Fig. 2E). Therefore, CYC1 silencing caused severe mitochondrial distress. Collectively, these results suggested that CYC1 silencing inhibited growth in OS cells via increasing apoptosis and damaging energy metabolism.

CYC1 silencing enhances TRAIL-induced Cytc release, caspase-9 activation, and apoptosis in OS cells

To find out whether CYC1 silencing sensitizes OS cells to TRAIL-induced apoptosis, we studied the effects of TRAIL alone or in combination with CYC1 silencing. Our results showed that TRAIL $(0-100 \mathrm{ng} / \mathrm{ml})$ alone dose-dependently induced apoptosis in 143B and MG-63 cells. Importantly, the percent of apoptotic cells increased further in 143B cells transfected with Lv-CYC1 shRNA (Fig. 3A). Similar effects were observed in MG-63 cells. In addition, treatment with TRAIL at $10 \mathrm{ng} / \mathrm{ml}$ triggered greater PARP cleavage in Lv-CYC1 shRNAtransfected 143B and MG-63 cells than control with TRAIL alone (Fig. 3B). Therefore, our results demonstrated that CYC1 silencing potentiates TRAIL-induced caspase activation and apoptosis in OS cells.

CYC1 silencing enhances TRAIL-induced Cytc release and caspase-9 activation in OS cells

A key step in mitochondria-dependent apoptotic pathway involves the release of Cytc into the cytosol and subsequent activation of caspase-9 [15]. To look into the mechanisms by which CYC1 silencing enhances TRAIL-induced apoptosis in OS cells, we assessed Cytc release and caspase- 9 activity. We found that CYC1 silencing alone slightly increased Cytc release but had no effect on the activity of caspase-9 in 143B and MG-63 cells (Fig. 4A, 4B). Meanwhile, 


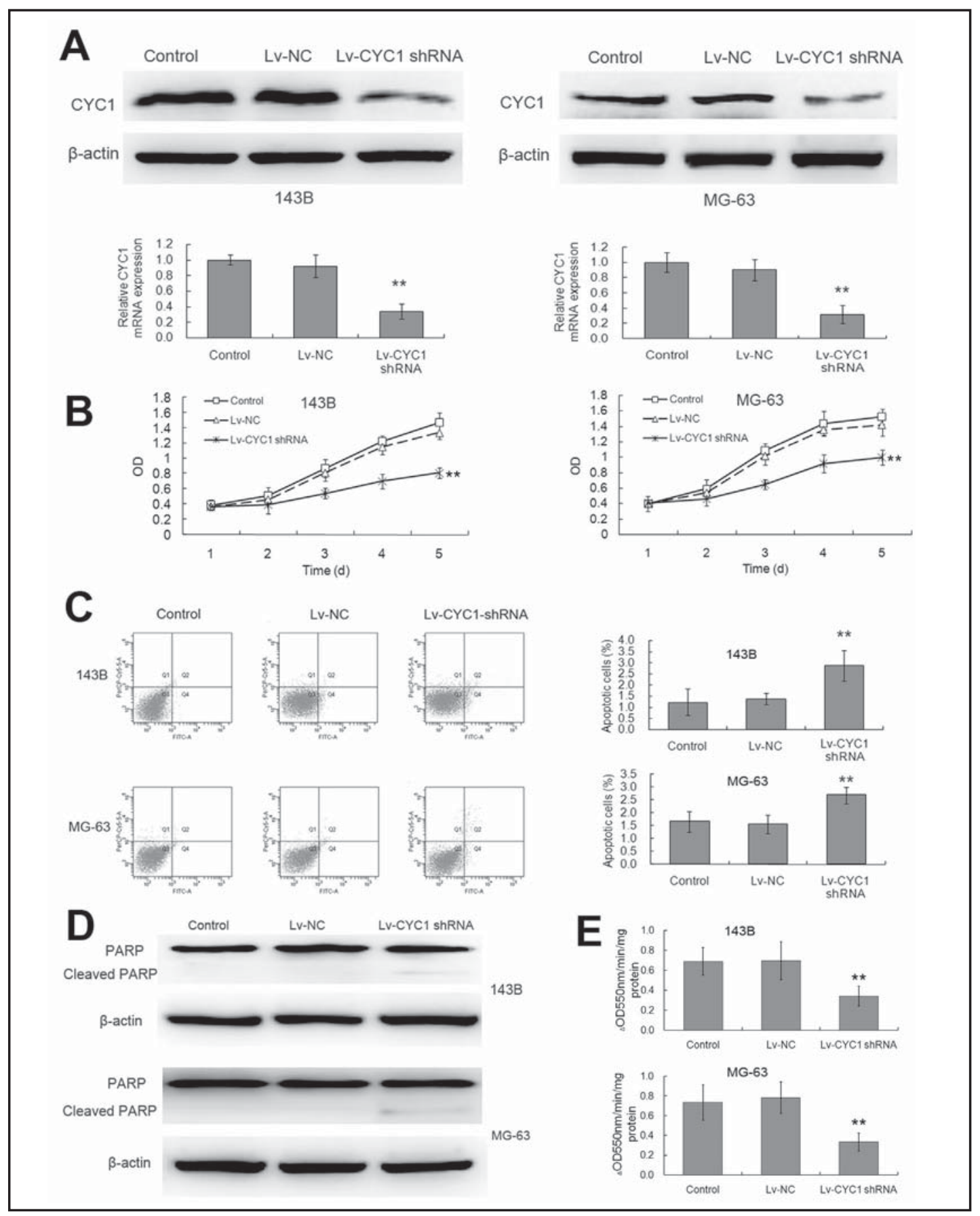

Fig. 2. CYC1 silencing inhibits growth and induces apoptosis in OS cells. 143B and MG-63 cells were transfected with control, Lv-NC, or Lv-CYC1 shRNA as described in materials and methods. (A) The protein and mRNA expression of CYC1 in transfected cells by western blot and real-time PCR, respectively. B) Proliferation of transfected cells by the MTT assay. (C) Apoptosis of transfected cells by annexin V-FITC/PI double-staining flow cytometry. (D) Levels of PARP and cleaved PARP in transfected cells by western blot analysis. (E) Complex III activity in transfected cells. Data are expressed as means \pm SD. ${ }^{* *} p<0.01$ compared with control.

treatment with $10 \mathrm{ng} / \mathrm{ml}$ TRAIL for $72 \mathrm{~h}$ resulted in moderate increases in Cytc release and caspase- 9 activity. Importantly, the combination of CYC1 silencing and TRAIL triggered significantly greater Cytc release and caspase- 9 activation in OS cells compared with either 
A

TRAlL (ng/ml) $\quad 0$
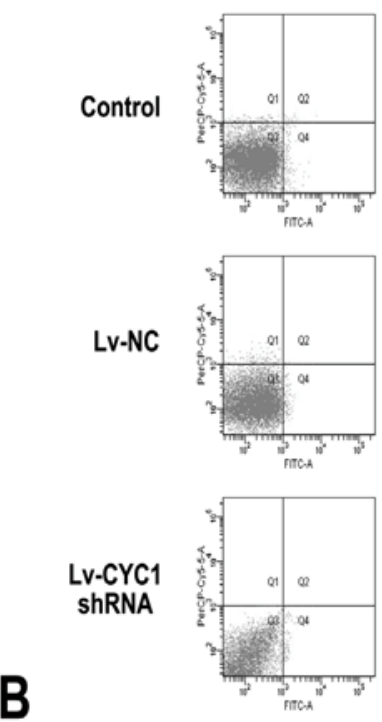

1
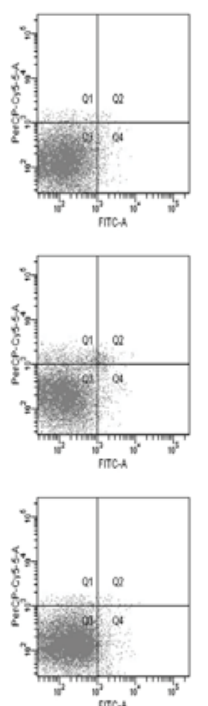

10
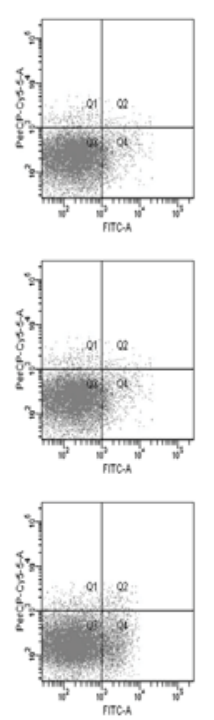

50
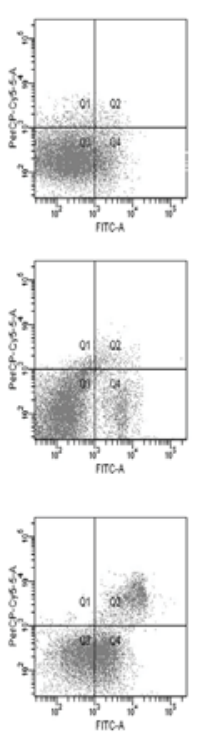

100
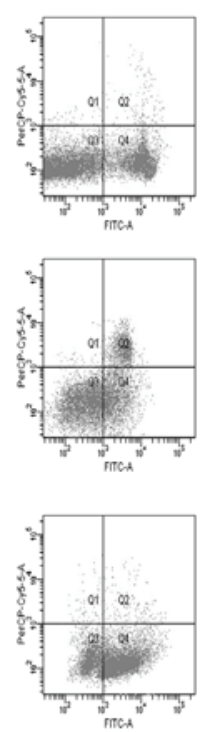
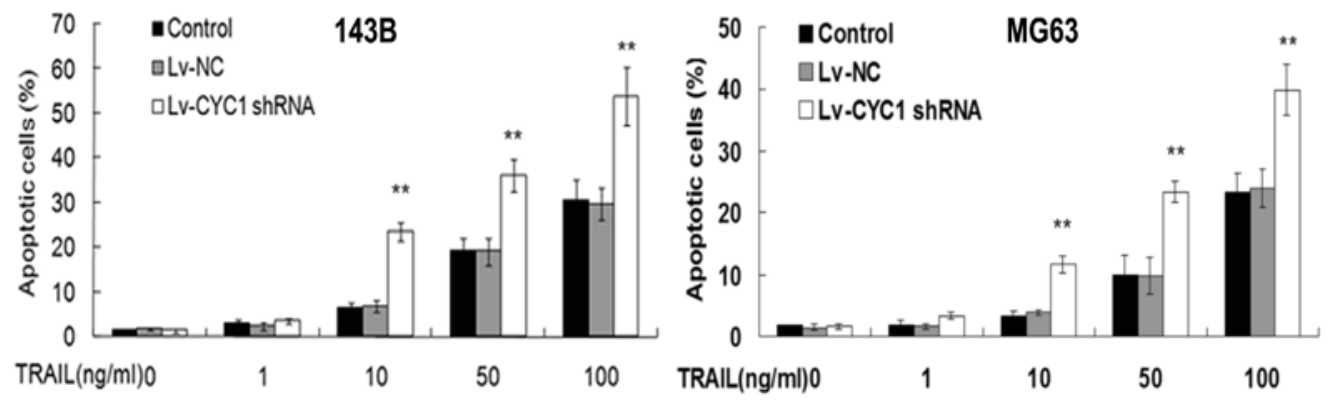

C

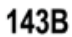

MG63

Control LV-NC LV-CYC1 shRNA Control LV-NC Lv-CYC1 shRNA Control Lv-NC LV-CYC1 shRNA Control LV-NC LV-CYC1 shRNA

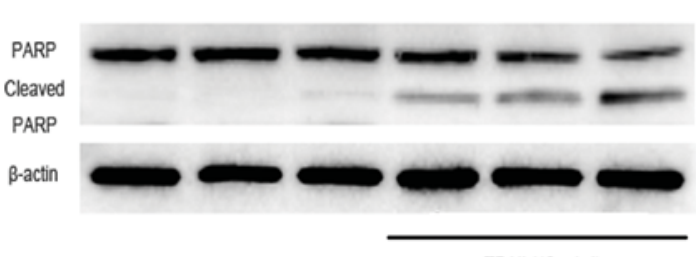

TRAIl(10ng/ml)

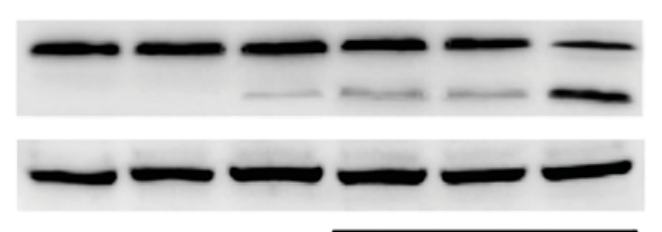

TRAIL(10ng/ml)

Fig. 3. CYC1 silencing sensitizes OS cells to apoptotic induction by TRAIL. 143B and MG-63 cells transfected with control, Lv-NC, or Lv-CYC1 shRNA were treated with TRAIL (1, 10, 50, $100 \mathrm{ng} / \mathrm{ml}$ ) for $72 \mathrm{~h}$. (A) The percent of apoptotic cells by annexin V-FITC/PI double-staining flow cytometry. (B) Levels of PARP and cleaved PARP by western blot analysis. Data are expressed as means \pm SD. ${ }^{* *} \mathrm{p}<0.01$ compared with control.

CYC1 silencing or TRAIL alone. These effects of CYC1 silencing were blocked by the caspase 3 inhibitor Z-DEVD-FMK (Fig. 4A and 4B). It has been reported that caspase-3 amplifies Cytc release and mitochondria fragmentation by cleaving CYC1 during mitochondria-dependent apoptosis [11]. In the mitochondrial electron transport chain, CYC1 directly interacts and stabilizes Cytc. It is believed that CYC1 cleavage by caspase-3 disrupts its physical interaction with Cytc, resulting in increased concentration of free Cytc and consequent Cytc release. We 


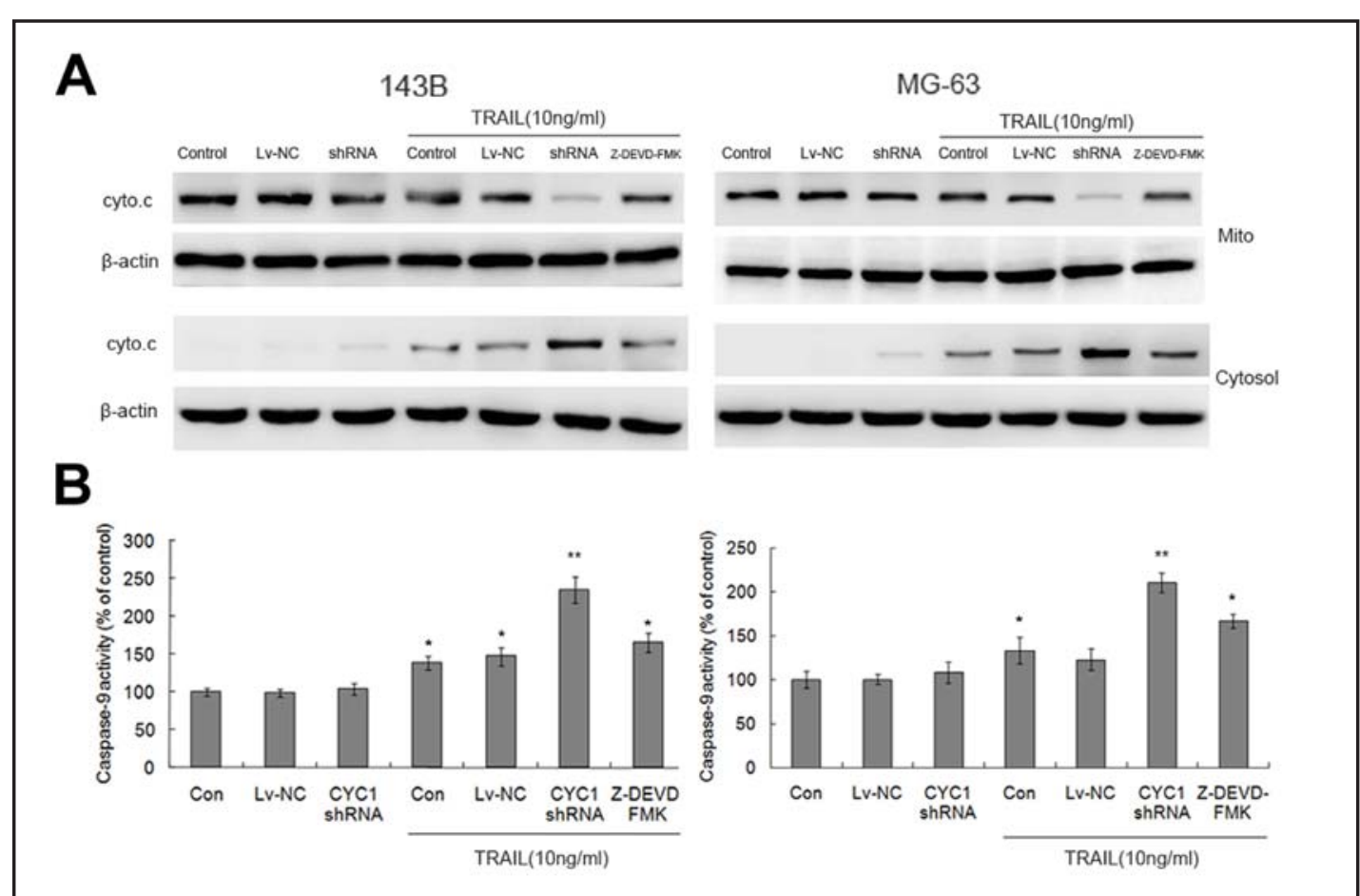

Fig. 4. CYC1 silencing enhances TRAIL-induced Cytc release and caspase-9 activation in OS cells. 143B and MG-63 cells transfected with control, Lv-NC, or Lv-CYC1 shRNA were treated with or without $10 \mathrm{ng} / \mathrm{ml}$ TRAIL for $72 \mathrm{~h}$. Additionally, cells transfected with Lv-CYC1 shRNA were incubated with $10 \mathrm{ng} / \mathrm{ml}$ TRAIL in the presence of the caspase-3 inhibitor Z-DEVD-FMK $(100 \mu \mathrm{M})$ for $72 \mathrm{~h}$. (A) The level of cytosolic Cytc by western blot analysis. (B) Caspase- 9 activity. Data are expressed as means \pm SD. ${ }^{*} p<0.05,{ }^{* *} p<0.01$ compared with control without TRAIL.

think that CYC1 silencing likely promotes Cytc release via similar mechanisms. Our results also suggested that blocking CYC1 cleavage with caspase-3 inhibitors might compensate for the loss of CYC1 function caused by reduced expression.

\section{CYC1 silencing sensitizes OS tumors to growth inhibition and apoptotic induction by TRAIL} in vivo

To investigate the relevance of our in vitro findings to OS tumorigenesis in vivo, we monitored the growth of tumors derived from 143B cells transfected with control, Lv-NC, or Lv-CYC1 shRNA in nu/nu nude mice. Consistent with our in vitro findings, tumors derived from Lv-CYC1 shRNA-transfected 143B cells grew at a much slower rate than those derived from control- or Lv-NC-transfected cells, as reflected in significantly smaller tumor volume ( $\mathrm{p}<0.05$ ) (Fig. 5A, 5B). Meanwhile, treatment with $10 \mathrm{mg} / \mathrm{kg}$ TRAIL every three days for six weeks only slightly inhibited growth of tumors derived from control- or Lv-NC-transfected 143B cells, indicating that these tumors were resistant to TRAIL in vivo. Importantly, we found that tumors derived from Lv-CYC1 shRNA-transfected 143B cells were extremely sensitive to TRAIL. Growth of these tumors was almost completely inhibited by TRAIL administration throughout the six-week treatment period (Fig. 5A, 5B). After the treatment was completed, we assessed the level of apoptosis in tumor tissues based on detection of fragmented DNA by the TUNEL assay. We found that tumors derived from Lv-CYC1 shRNA-transfected 143B cells had higher levels of apoptosis compared with those derived from control- or Lv-NCtransfected cells $(\mathrm{p}<0.05)$ (Fig. 5C). TRAIL treatment alone did not induce apoptosis in tumor tissues; however, the combination of CYC1 silencing and TRAIL administration resulted in greatly increased apoptosis ( $\mathrm{p}<0.01$ ) (Fig. 5C). Thus, the level of apoptosis in tumor tissues was in alignment with the rate of tumor growth. 


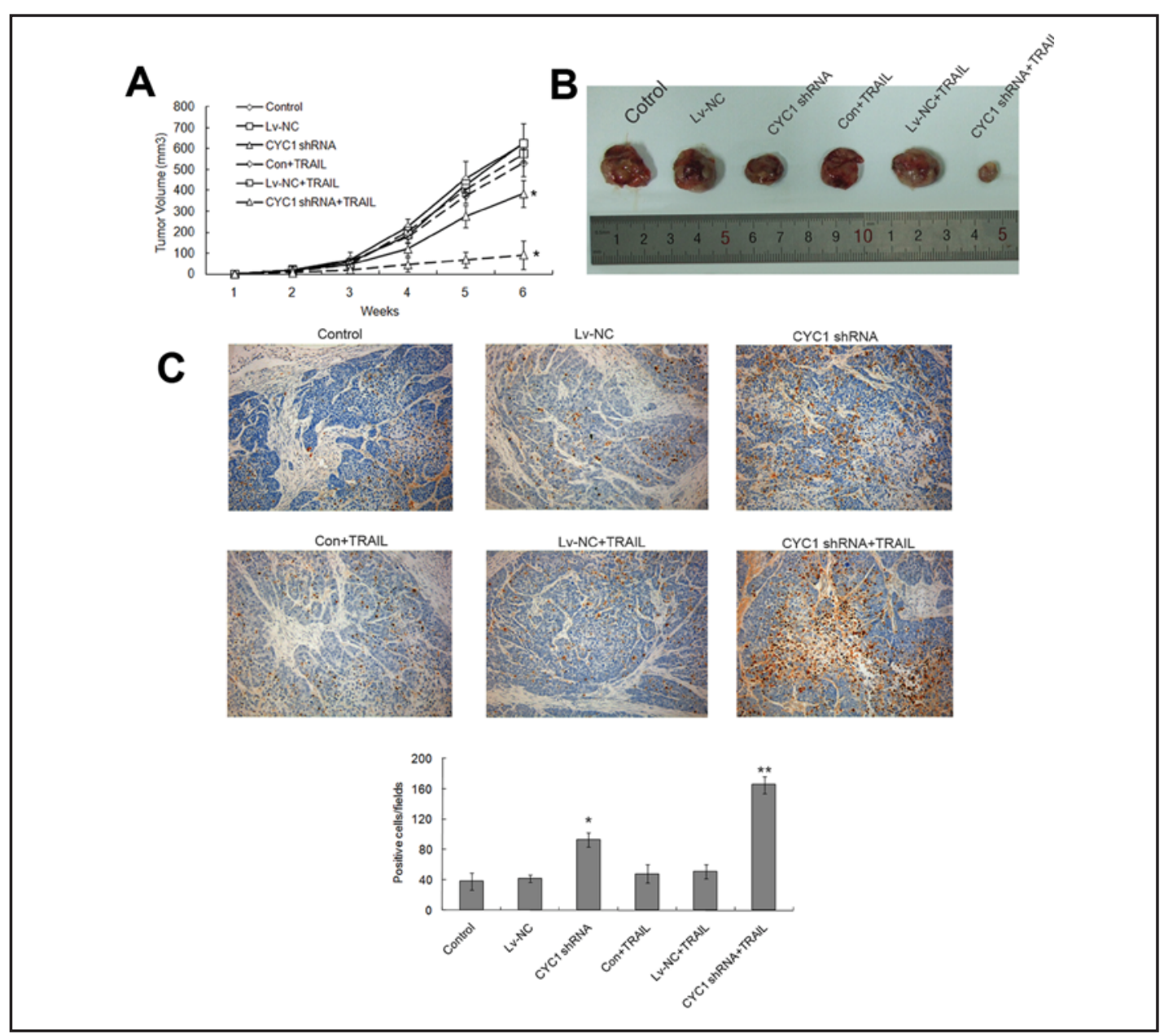

Fig. 5. CYC1 silencing sensitizes OS tumors to growth inhibition and apoptotic induction by TRAIL in vivo. 143B cells transfected with control, Lv-NC, or Lv-CYC1 shRNA were injected subcutaneously into the flank of nude mice. Mice $(n=6)$ received a single intravenous injection of $10 \mathrm{mg} / \mathrm{kg}$ TRAIL every three days for six weeks. (A) Tumor volume measured weekly. (B) Tumor size at the end of the six-week treatment. (C) The level of apoptosis in tumor tissues by the TUNNEL assay. Data are expressed as means \pm SD. ${ }^{*} p<0.05$, ${ }^{* *} \mathrm{p}<0.01$ compared with control without TRAIL.

\section{Discussion}

Diverse chemotherapeutic agents kill cancer cells by activating common apoptotic pathways. Inherent resistance to apoptosis underpins both tumorigenesis and drug resistance of many malignancies. Thus, new therapeutic strategies to overcome cancer cell's resistance to apoptosis are required for effective cancer treatment. TRAIL is a promising strategy since it induces targeted apoptosis in malignant cells without affecting normal cells [16]. However, a considerable number of cancer cells, especially some highly malignant tumors, are resistant to apoptotic induction by TRAIL, and require combination treatment with other chemotherapeutic agents [17]. Apoptosis can be induced by distinct but overlapping pathways. TRAIL induces cancer cell apoptosis via an "extrinsic", deathreceptor-mediated pathway. Numerous studies have shown that compounds stimulating the "intrinsic", mitochondria-dependent apoptotic pathway potentiate tumors to apoptotic induction by TRAIL [18-21]. Thus, mitochondrion is proposed as a direct target for the development of effective TRAIL sensitizers [19]. 


\section{Cellular Physiology and Biochemistry}

Cell Physiol Biochem 2014;34:2070-2080

\begin{tabular}{l|l}
\hline DOI: $10.1159 / 000366402$ & (C) 2014 S. Karger AG, Basel
\end{tabular}

Published online: November 28, 2014

www.karger.com/cpb

Osteosarcoma (OS) is an aggressive malignancy with poor prognosis. It has been reported that inherent resistance to apoptosis is one of the mechanisms by which OS cells escape therapeutic control $[22,23]$. Most tumor cells are sensitive to apoptosis induced by TRAIL; however, many osteosarcomas are resistant to TRAIL [24, 25]. Intriguingly, our previous study showed that cytochrome $\mathrm{c} 1$ (CYC1), an indispensible protein component of the mitochondrial electron transport chain, is found at elevated concentration in the serum of OS patients, and the concentration drops after surgical resection [9]. CYC1 has also been found to be overexpressed in nasopharyngeal carcinoma (NPC) tissues and cell lines [10], implying that CYC1 might play a role in the development of specific types of cancer. Interestingly, a recent study identified CYC1 as a potential gate-keeper against mitochondriadependent apoptosis. Its cleavage by caspase- 3 amplifies death stimuli-induced mitochondria dysfunction, Cytc release, and apoptosis [11]. Therefore, we speculated that suppression of CYC1 expression in OS cells might stimulate the "intrinsic", mitochondria-dependent apoptosis pathway, and thereby potentiate OS cells to apoptotic induction by TRAIL.

In the present study, we first demonstrated that CYC1 is overexpressed in human OS tumor tissues and cell lines, which agrees with previous reports [9]. Our subsequent in vitro studies demonstrated that CYC1 silencing by shRNA transfection inhibited growth in human OS cell lines. Further studies showed that CYC1 silencing had a slight effect on apoptosis, but significantly reduced mitochondrial complex III activity in OS cell lines. Similar to our in vitro findings, CYC1 silencing curbed OS tumor growth in vivo. We subsequently tested the effects of TRAIL, either alone or in combination with CYC1 silencing. The OS cell lines 143B and MG-63 showed moderate sensitivity to apoptotic induction by TRAIL in vitro; however, tumors derived from 143B cells were resistant to TRAIL in vivo. We found that CYC1 silencing significantly enhanced TRAIL-induced Cytc release, caspase activation, and apoptosis in OS cells in vitro, and sensitized OS tumors to apoptotic induction by TRAIL in vivo, resulting in almost complete inhibition of tumor growth through a six-week treatment period.

A variety of agents to sensitize OS to TRAIL-induced apoptosis have been proposed [26]. In this study, we showed that CYC1 downregulation effectively potentiates OS to apoptotic induction by TRAIL in vitro and in vivo, and these effects are mediated by activation of the mitochondria-dependent apoptotic pathway. Since CYC1 physically interacts with Cytc, CYC1 silencing may destabilize its interaction with Cytc. A small amount of activated caspase-3 may further disrupt the interaction and lead to an increase in free Cytc concentration and consequent Cytc release. Although the mechanisms are not fully elucidated, our results demonstrate that modulation of CYC1 may be an effective strategy to potentiate OS to apoptotic induction by TRAIL.

\section{Acknowledgement}

This work was supported by National Science Foundation(81202116), "Qimingxing" talent project of Shanghai science and technology commission (13QA1403000), Science Foundation of Zhejiang Province(NO.LY13H060005), General Research Plan B of Zhejiang province (N0.2012KYB213) and Shaoxing Science Project (No.2013B70081).

\section{References}

1 Mirabello L, Troisi RJ, Savage SA: International osteosarcoma incidence patterns in children and adolescents, middle ages and elderly persons. Int J Cancer 2009;125:229-234.

$\longrightarrow 2$ Jaffe N: Adjuvant chemotherapy in osteosarcoma: An odyssey of rejection and vindication. Cancer Treat Res 2009;152:219-237.

-3 Chou AJ, Gorlick R: Chemotherapy resistance in osteosarcoma: Current challenges and future directions. Expert Rev Anticancer Ther 2006;6:1075-1085.

4 Gorlick R, Janeway K, Lessnick S, Randall RL, Marina N: Children's oncology group's 2013 blueprint for research: Bone tumors. Pediatr Blood Cancer 2013;60:1009-1015. 


\section{Cellular Physiology and Biochemistry}

Cell Physiol Biochem 2014;34:2070-2080

\begin{tabular}{l|l}
\hline DOI: $10.1159 / 000366402$ & (C) 2014 S. Karger AG, Basel
\end{tabular}

Published online: November 28, $2014 \quad$ www.karger.com/cpb

Li et al.: CYC1 Promoting Osteosarcoma Apoptosis

5 Holoch PA, Griffith TS: Tnf-related apoptosis-inducing ligand (trail): A new path to anti-cancer therapies. Eur J Pharmacol 2009;625:63-72.

6 Mirandola P, Sponzilli I, Gobbi G, Marmiroli S, Rinaldi L, Binazzi R, Piccari GG, Ramazzotti G, Gaboardi GC, Cocco L, Vitale M: Anticancer agents sensitize osteosarcoma cells to tnf-related apoptosis-inducing ligand downmodulating iap family proteins. Int J Oncol 2006;28:127-133.

7 Hotta T, Suzuki H, Nagai S, Yamamoto K, Imakiire A, Takada E, Itoh M, Mizuguchi J: Chemotherapeutic agents sensitize sarcoma cell lines to tumor necrosis factor-related apoptosis-inducing ligand-induced caspase-8 activation, apoptosis and loss of mitochondrial membrane potential. J Orthop Res 2003;21:949957.

8 Crofts AR: The cytochrome bc1 complex: Function in the context of structure. Annu Rev Physiol 2004;66:689-733.

-9 Li G, Zhang W, Zeng H, Chen L, Wang W, Liu J, Zhang Z, Cai Z: An integrative multi-platform analysis for discovering biomarkers of osteosarcoma. BMC Cancer 2009;9:150.

10 Liu Z, Wang HY, Long J, Fang WY: [construction of a lentivirus interfering vector targeting cyc1 and its interfering efficiency in nasopharyngeal carcinoma cells]. Nan Fang Yi Ke Da Xue Xue Bao 2010;30:26292632.

11 Zhu Y, Li M, Wang X, Jin H, Liu S, Xu J, Chen Q: Caspase cleavage of cytochrome c1 disrupts mitochondrial function and enhances cytochrome c release. Cell Res 2012;22:127-141.

-12 Mohseny AB, Machado I, Cai Y, Schaefer KL, Serra M, Hogendoorn PC, Llombart-Bosch A, Cleton-Jansen AM: Functional characterization of osteosarcoma cell lines provides representative models to study the human disease. Lab Invest 2011;91:1195-1205.

13 Shou Y, Li L, Prabhakaran K, Borowitz JL, Isom GE: Calcineurin-mediated bad translocation regulates cyanide-induced neuronal apoptosis. Biochem J 2004;379:805-813.

-14 Eliseev RA, Zuscik MJ, Schwarz EM, O'Keefe RJ, Drissi H, Rosier RN: Increased radiation-induced apoptosis of saos2 cells via inhibition of nfkappab: A role for c-jun n-terminal kinase. J Cell Biochem 2005;96:12621273.

15 Kroemer G: Mitochondrial control of apoptosis: An introduction. Biochem Biophys Res Commun 2003;304:433-435.

16 Ashkenazi A, Pai RC, Fong S, Leung S, Lawrence DA, Marsters SA, Blackie C, Chang L, McMurtrey AE, Hebert A, DeForge L, Koumenis IL, Lewis D, Harris L, Bussiere J, Koeppen H, Shahrokh Z, Schwall RH: Safety and antitumor activity of recombinant soluble apo2 ligand. J Clin Invest 1999;104:155-162.

17 Rowinsky EK: Targeted induction of apoptosis in cancer management: The emerging role of tumor necrosis factor-related apoptosis-inducing ligand receptor activating agents. J Clin Oncol 2005;23:9394-9407.

-18 Ziauddin MF, Yeow WS, Maxhimer JB, Baras A, Chua A, Reddy RM, Tsai W, Cole GW Jr, Schrump DS, Nguyen DM: Valproic acid, an antiepileptic drug with histone deacetylase inhibitory activity, potentiates the cytotoxic effect of apo2l/trail on cultured thoracic cancer cells through mitochondria-dependent caspase activation. Neoplasia 2006;8:446-457.

-19 Nguyen DM, Yeow WS, Ziauddin MF, Baras A, Tsai W, Reddy RM, Chua A, Cole GWJr, Schrump DS: The essential role of the mitochondria-dependent death-signaling cascade in chemotherapy-induced potentiation of apo2l/trail cytotoxicity in cultured thoracic cancer cells: Amplified caspase 8 is indispensable for combination-mediated massive cell death. Cancer J 2006;12:257-273.

20 Shamimi-Noori S, Yeow WS, Ziauddin MF, Xin H, Tran TL, Xie J, Loehfelm A, Patel P, Yang J, Schrump DS, Fang BL, Nguyen DM: Cisplatin enhances the antitumor effect of tumor necrosis factor-related apoptosisinducing ligand gene therapy via recruitment of the mitochondria-dependent death signaling pathway. Cancer Gene Ther 2008;15:356-370.

21 Yang L, Lan C, Fang Y, Zhang Y, Wang J, Guo J, Wan S, Yang S, Wang R, Fang D: Sodium nitroprusside (snp) sensitizes human gastric cancer cells to trail-induced apoptosis. Int Immunopharmacol 2013;17:383-389.

22 Tsagaraki I, Tsilibary EC, Tzinia AK: Timp-1 interaction with alphavbeta3 integrin confers resistance to human osteosarcoma cell line mg-63 against tnf-alpha-induced apoptosis. Cell Tissue Res 2010;342:87-96.

23 Sollazzo V, Galasso M, Volinia S, Carinci F: Prion proteins (prnp and prnd) are over-expressed in osteosarcoma. J Orthop Res 2012;30:1004-1012.

-24 Sun J, Fu ZM, Fang CQ, Li JH: Induction of apoptosis in osteogenic sarcoma cells by combination of tumor necrosis factor-related apoptosis inducing ligand and chemotherapeutic agents. Chin Med J (Engl) 2007;120:400-404.

25 Evdokiou A, Bouralexis S, Atkins GJ, Chai F, Hay S, Clayer M, Findlay DM: Chemotherapeutic agents sensitize osteogenic sarcoma cells, but not normal human bone cells, to apo2l/trail-induced apoptosis. Int J Cancer 2002;99:491-504.

26 Gasparini C, Vecchi Brumatti L, Monasta L, Zauli G: Trail-based therapeutic approaches for the treatment of pediatric malignancies. Curr Med Chem 2013;20:2254-2271. 